\title{
A Wideband Low-Profile All-Metal Cavity Slot Antenna with Filtering Performance for Space-Borne SAR Applications
}

\author{
Xiaochuan Fang, Wei Wang, Qi Luo, Member, IEEE, Steven Gao, Senior Member, IEEE, Lichun Sun, \\ Wei Guan and Hongtao Zhang
}

\begin{abstract}
This letter presents a wideband All-metal cavity slot antenna for space-borne SAR applications which achieves the filtering performance simultaneously. The proposed antenna consists of a metal cavity and several radiation slots. It was fabricated by aluminium through milling and welding process which is suitable for the space environment. Benefiting from multi resonances of the cavity and slots, the antenna obtains a wide bandwidth. An 8x12 antenna array is designed, fabricated and measured for verification. The measured impedance bandwidth of $51 \%$ from $7.2 \mathrm{GHz}$ to $12.2 \mathrm{GHz}$ for $\mathrm{S11}<-10 \mathrm{~dB}$ is achieved. Furthermore, it shows good filtering performance of more than $20 \mathrm{~dB}$ suppression level in the out-band comparing to the operating band which suppresses the unwanted interferences and contributes to the good EMC design. The cavity has a very low profile of $2.8 \mathrm{~mm}$, i.e. $0.067 \lambda$ at the lowest operating frequency.
\end{abstract}

Index Terms-Wideband, Low-profile, All-metal cavity, Slot antenna, Filtering, Space-Borne SAR.

\section{INTRODUCTION}

Synthetic aperture radar (SAR) is a novel type of radar system that has good environmental adaptability, high reliability, long working duration, and is well accepted in many different areas such as environmental mapping and protection, military observation, traffic controlling, disaster monitoring, etc. As one of the most important components, antenna design for SAR always attracts much research interests. Reflector, microstrip patch and waveguide slot antennas array are usually chosen for SAR system [1-4]. There many strict requirements to the antennas that used for SAR especially space-borne SAR, such as compact size, lightweight, high structural strength and good heat dissipation, as well as high efficiency and the ability for beam scanning. All-metal antennas are recently gain interests in outer-space applications, which can be expected to withstand harsh temperature conditions and radiation levels existed in such environments [5], whereas limited scanning

Manuscript submitted on December 14, 2018. This work was supported by the National Natural Science Foundation of China (NSFC) under Grant [61671416].

X. C. Fang is with the School of Information and Communication Engineering, University of Electronic Science and Technology of China, Chengdu 611731, P. R. China (fang.x.c@163.com).

W. Wang, L. C. Sun, W. Guan and Hongtao Zhang are with the East China Research Institute of Electronic Engineering, Hefei 230088, Anhui, P. R. China (shu00ww@163.com).

Q. Luo and S. Gao are with the School of Engineering and Digital Arts, University of Kent, Canterbury, UK. E-mail (qiluo@ieee.org; s.gao@kent.ac.uk). active phased arrays currently play important role in space-borne SAR system to meet these demands.

The high-resolution SAR system requires 2-D wide angle scanning active phased antenna array [6-7]. This means that the antenna array must achieve broad bandwidth, for example, $3 \mathrm{GHz}$ bandwidth is needed for $0.1 \mathrm{~m}$ resolution, and a T/R module only terminates with one or two radiating elements for the wide-angle scanning. The loss of the feed network is significantly reduced comparing with that of the multi-element subarray. The multilayer microstrip patch antenna has wide bandwidth, lightweight and low-profile. However, its poor heat conduction ability increases the difficulty of the thermal control measure for the active phased array. The profile of the Vivaldi antenna is high and face the same problem as the microstrip antenna array.

Meanwhile, the electromagnetic compatibility (EMC) becomes a prominent problem as the SAR system crowded into a congested space with other systems, such as data transmission, navigation, and telemetry et al. The filtering antenna is a good candidate for the front-end of the system. The filter can be integrated into the feed line of an antenna array [8-10]. For an antenna element, many designers choose to use the unintegrated structures, which means using antenna itself as the filter which can avoid the use of extra filtering structures and reduce the impedance mismatching [11-14]. However, most of the reported designs are based on a single layer or multilayer patch or an SIW as the substrate. They are not good candidates for the space-borne active phased array applications considering the thermal control, long life and robust.

Based on our previous research [15-16], a wideband all-metal cavity antenna with a good filtering characteristic is presented in this letter. The antenna consists of a rectangular metal cavity, an H-shaped slot and a pair of the C-shaped slot. It uses a simple structure to realize a wide bandwidth and a stable passband performance. The metal material is good for the heat dissipation from the $T / R$ module. The multi-column and multi-row element array was fabricated in an integral component, achieving a good structural strength because of its grid construction. The parameters have been analyzed, and the simulation, as well as the measurement results of an $8 \times 12$ antenna array, are presented.

\section{Antenna Design}

\section{A: Antenna configuration}

Fig.1 illustrates the proposed antenna, which consists of a rectangular metal cavity, a metal matching block, an H-shaped 
slot and a pair of C-shaped slots. The slots are milled in the top layer of the cavity, acting as the radiator. The antenna is fed by an SMA connector which is easy to interface with a T/R module. The matching metal block is stuck on the down face of the top layer between the $\mathrm{H}$-shaped slot and the $\mathrm{C}$-shaped slot, as shown in Fig.1. The inner conductor of the SMA is inserted into a hole milled in the block.

Some essential dimensions of the antenna are $L=21.3 \mathrm{~mm}$, $W=15.6 \mathrm{~mm}, H=2.8 \mathrm{~mm}$ (height of the cavity), $L_{\mathrm{slot}}=4 \mathrm{~mm}$, $W_{\text {slot }}=7 \mathrm{~mm}$, and $L_{\text {cut }}=1.5 \mathrm{~mm}$. These parameters play important roles in determining the antenna performance. Noticed that the antenna cavity has a very low-profile of $0.067 \lambda$ at the lowest operating frequency, which allow the antenna suitable for different application scenarios and array conformation. The height of the whole antenna is $3.8 \mathrm{~mm}$ which contains of the contains the height of the top and bottom metal cavity wall, each of them is $0.5 \mathrm{~mm}$.

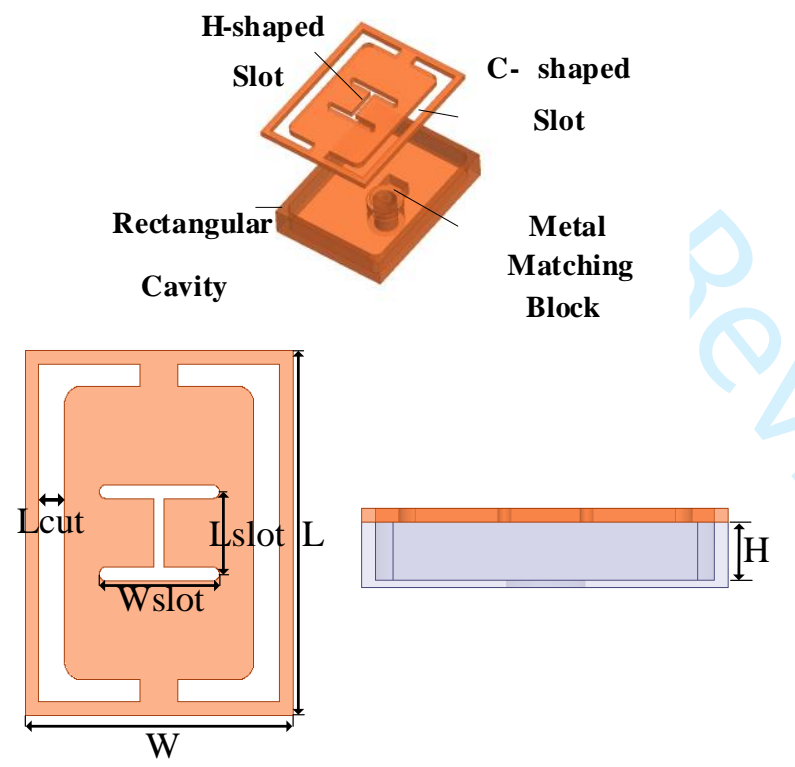

Fig.1. Antenna constituent details and configurations.

\section{B: Wideband impedance matching and filtering}

The rectangular cavity is used as the antenna substrate, so the developed antenna can be kept as a single independent module. This provides a controllable continuous impedance bandwidth, which ensures the stable radiation pattern and maximizes the antenna gain. The feeding method of the antenna excites the standard waveguide operating mode, which can be used to match the impedance of the filtering bandwidth. The currents on the top layer of the cavity radiate from these two different slots independently thus generate electromagnetic resonance at different frequencies, which contributes to the wide bandwidth. A matching block is introduced to provide extra inductance in the proposed design. The comparison of the S11 with and without the matching block (Fig.2(a)) is shown in Fig.2(b).

The filtering operation of this antenna has also been studied. It is evident that the slots can be equivalent as a capacitance and the rectangular cavity can be equivalent as an inductance. Thus, Fig.3 illustrates the simplified equivalent circuit of the antenna filtering system, where the effect of each part of the antenna is represented by a lumped element. It is concluded that the component that determines the lower-band radiation null frequency is the C-shaped slots, which is justified by the comparison of the simulated realized gain of the antenna with and without C-shaped slots (structure in Fig.4(a-b)). As shown in Fig.4(c), the lower-band realized gain is monotone increasing when the antenna has no C-shaped slot. In this case, there is no radiation null frequency while the upper-band radiation null frequency point is almost unaffected. Similarly, the upper-band radiation null frequency is controlled by the H-slot, as shown in Fig.4(f), and it cannot influence the lower-band. It can be concluded that the C-shaped slots and $\mathrm{H}$-shaped slot control their resonance response independently.

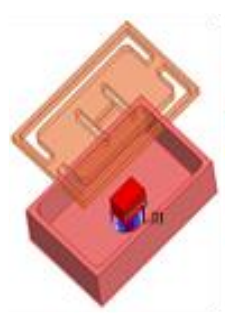

(a)

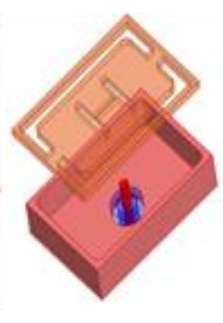

(b)

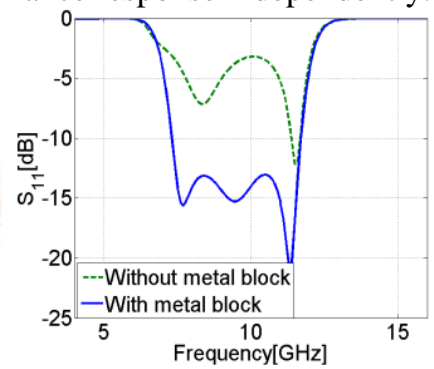

(c)
Fig.2. Antenna configuration (a) with metal block (b) without the metal block (c) impedance bandwidth comparison with and without the metal block.

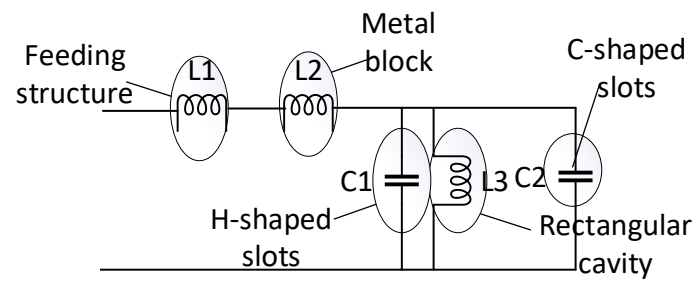

Fig.3. The equivalent circuit of the antenna filtering system.

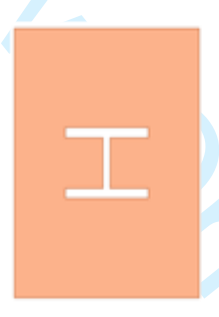

(a)

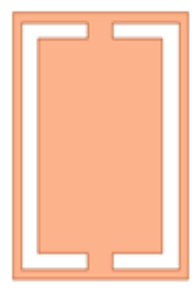

(d)

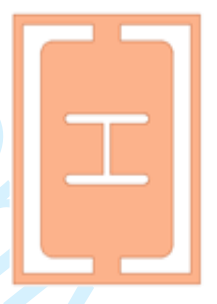

(b)

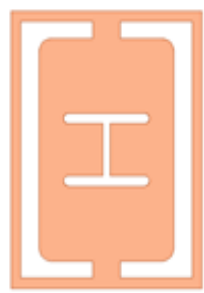

(e)

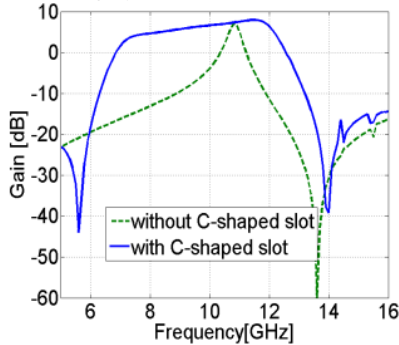

(c)

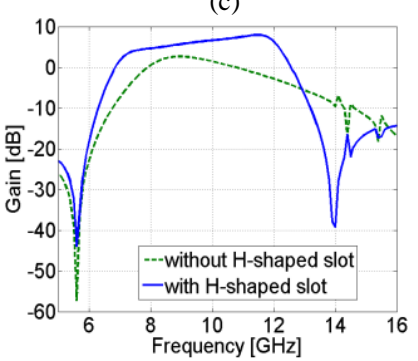

(f)
Fig.4. Antenna metal patch (a) without C-shaped slots (b) with C-shaped slots. (c) radiation gain comparison with and without $\mathrm{C}$-shaped slot (d) without $\mathrm{H}$-shaped slot (e) with the $\mathrm{H}$-shaped slot. (f) radiation gain comparison with and without I-shaped slots.

\section{C: Parametric study}

To optimize the antenna performance, some important parameters are studied. Firstly, as the most important parameters, the dimensions of $\mathrm{C}$-shaped and $\mathrm{H}$-shaped slots has been analyzed. Fig. 5(a) and (b) show the impedance bandwidth that influenced by $\mathrm{C}$-shaped slot width $L_{\text {cut }}$ and $\mathrm{H}$-shaped slot 
length $W_{\text {slot. }}$ When the $L_{\text {cut }}$ is less than $1.5 \mathrm{~mm}$, the $S 11$ of the first and the second resonance point are lower but the passband deteriorated when the $L_{\text {cut }}$ decreases. As for $W_{\text {slot }}$, the return loss of the first and second resonance point have a contrary trend with the changing of its dimension so the $6.5 \mathrm{~mm}$ is chosen. Another length of the $\mathrm{H}$-shaped slot $L_{\text {slot }}$ was also been analyzed and have a similar effect as $W_{\text {slot }}$ so the simulation results are not presented .

The effects of these parameters on the radiation performance of the antenna are given in Fig.5 (c) and (d). The optimum values ensure a wide and flat passband and the filtering effect seems deteriorated when $L_{\text {cut }}$ is less than $1.5 \mathrm{~mm}$ and the $W_{\text {slot }}$ is more than $6.5 \mathrm{~mm}$ although filtering band was expanded. It is also worth to point out that the changing of the $L_{\text {cut }}$ and $W_{\text {slot }}$ can independently control the lower-band and upper-band radiation null frequency, which also verifies the above analysis. Meanwhile, the cavity height $H$ and the length of the metal block $L_{\mathrm{m}}$ has also been studied in Fig.5 (e) and (f). It shows that the return loss of the first and the second resonance point become better when the $H$ increase while the frequencies of such points become closer so that the best value was found at $H=2.8 \mathrm{~mm}$. A very interesting phenomenon studied from Fig.5 (f) and also from other dimensions of the metal block is that the change of these dimensions has very limited influence on antenna impedance performance.

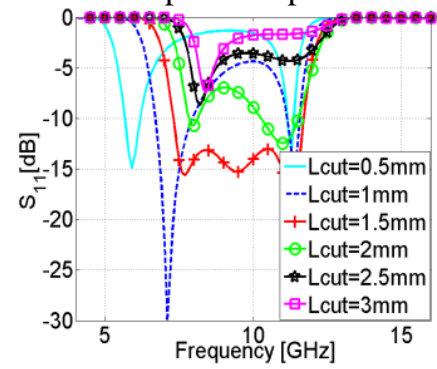

(a)

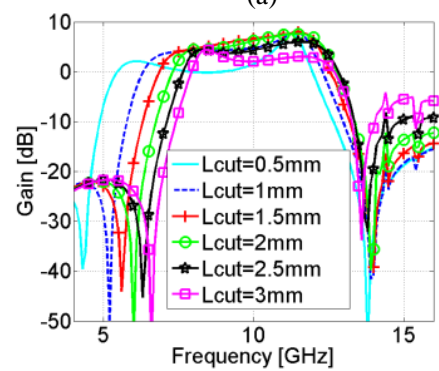

(c)

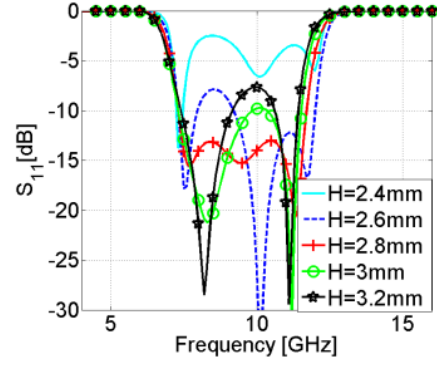

(e)

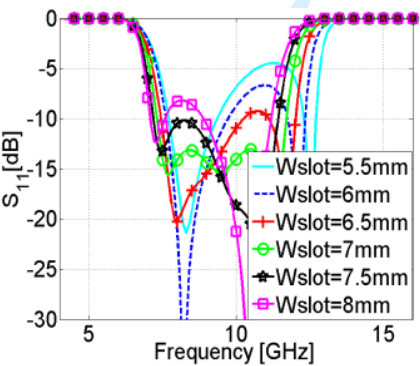

(b)

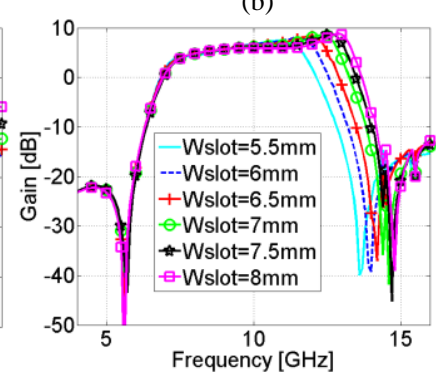

(d)

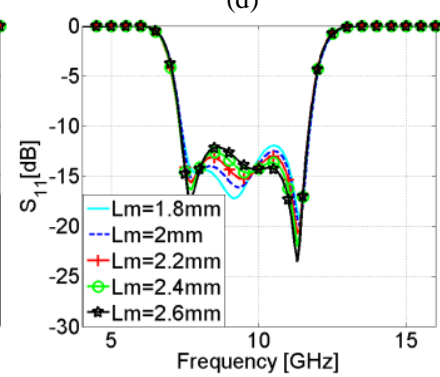

(f)
Fig. 5. Effect on (a) impedance bandwidth of C-shaped width $L_{\text {cut }}$ (b) impedance bandwidth of $\mathrm{H}$-shaped slot length $W_{\text {slot }}$ (c) radiation gain of C-shaped width $L_{\text {cut }}$ (d) radiation gain of $\mathrm{H}$-shaped slot length $W_{\text {slot }}$ (e) impedance bandwidth of cavity height $H$ (f) impedance bandwidth of metal block length $L_{\mathrm{m}}$

\section{MEASUREMENT Results AND Discussion}

Using the developed all-metal cavity slot element, an $8 \times 12$ elements array demonstrator for space-borne synthetic aperture radar (SAR) was fabricated. The antenna array was fabricated by a single metal material (see Fig. 6(a)), which provides good heat dissipation and high strength. Fig.6(b) shows the photo of the fabricated array prototype. The antenna filtering character is measured by a far-ranged UWB horn antenna so that it was expressed by a normalized S21. The radiation pattern of the antenna element was measured in a far-field chamber, whereas the radiation pattern of the antenna array was measured in a near-filed chamber to shows its beam scanning operation.

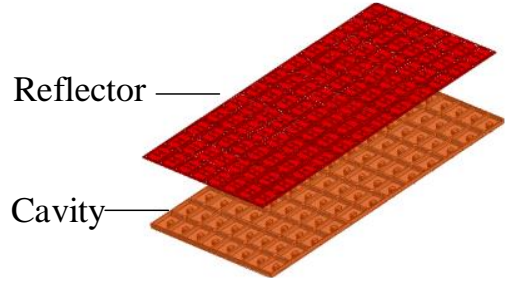

(a)

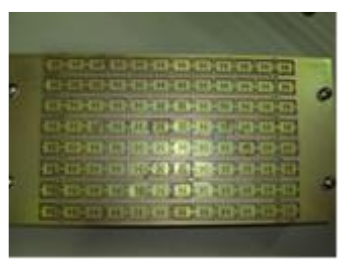

(b)
Fig.6. Fabricated Antenna array (a) geometry; (b) prototype.

Firstly, the return loss and normalized boresight gain of the antenna element is characterized. The measurements were performed by measuring one of the antenna elements in the center of the array. The simulated and measured antenna return loss is given in Fig. 7(a), showing that the simulated $\mathrm{S} 11<-10 \mathrm{~dB}$ is from $7.5 \mathrm{GHz}-11.5 \mathrm{GHz}$, representing $42 \%$ of fractional bandwidth. Outside the impedance bandwidth, there is a high roll-off rate. The measured return loss shows a wider impedance bandwidth with some frequency shifts, and the $10-\mathrm{dB}$ return loss is from $7.2 \mathrm{GHz}-12.2 \mathrm{GHz}$, about $51 \%$. Within this band, the measured return loss has a higher amplitude than the simulation results, which leads to some disagreement of the realized gain as shown in Fig.7(b). The simulated and measured normalized gain of the antenna element is shown in Fig.7(b), it contains the gain performance in passband and filter suppression off the passband. The figure was normalized at the standard of the peak value in order to observe the filtering features. As the figure indicates, two radiation nulls can be seen at $5.6 \mathrm{GHz}$ and $14 \mathrm{GHz}$. The simulation and measurement results show that the antenna realized gain have more than $20 \mathrm{~dB}$ suppression level in most operation bandwidth.

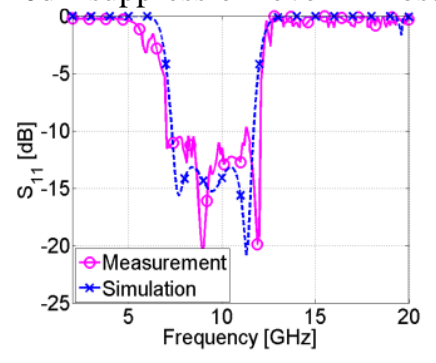

(a)

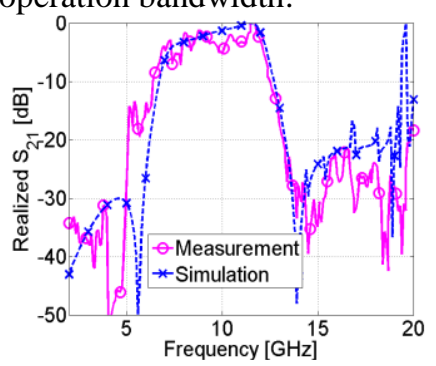

(b)
Fig.7. (a)Simulation and measurement return loss, (b)Simulation and Measurement Realized S21

The radiation patterns of this antenna in lower $(8 \mathrm{GHz})$, center $(10 \mathrm{GHz})$ and higher $(12 \mathrm{GHz})$ frequency are shown in Fig.8, which compares the simulation and measurement gains in all the operating bandwidth. It can be concluded that all the radiation patterns are nearly the same and the cross polarization 
has a more than $-40 \mathrm{~dB}$ lower than the primary polarization. Fig.8(a), (c) and (e) shows the radiation patterns in E(xoz)-plane while the Fig.8(b), (d) and (f) shows the radiation patterns in $\mathrm{H}(\mathrm{yoz})$-plane, which show that the antenna has the similar radiation patterns in these two principal planes. The measurement results also show that the gain of the antenna element is approximately $5 \mathrm{dBi}$ in the lower band and $7 \mathrm{dBi}$ in the upper band. The measured efficiency of the antenna element was higher than $85 \%$ in the operation band.

The external phase shifters are connected to the antennas and the radiation patterns of the array radiated at broadside and 30 degrees off the broadside were measured. The radiation patterns of the uniform excited antenna array at $8 \mathrm{GHz}, 10 \mathrm{GHz}$, and $12 \mathrm{GHz}$ in $\Theta=0$ and $\Theta=30^{\circ}$ are presented in Fig.9, showing that the antenna has stable radiation patterns in the required frequencies which meet the requirements of the SAR application.

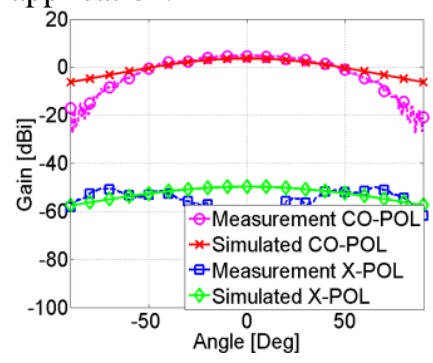

(a)

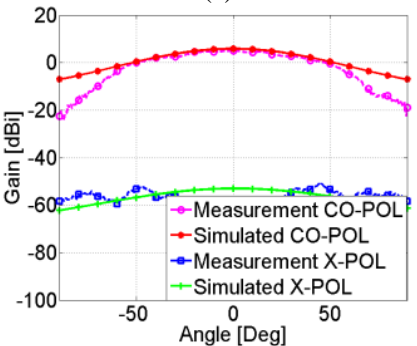

(c)

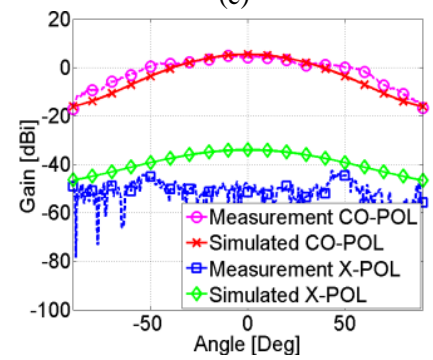

(e)

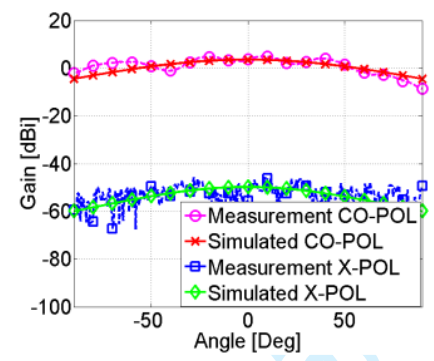

(b)

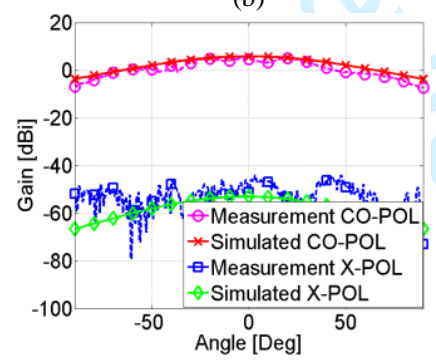

(d)

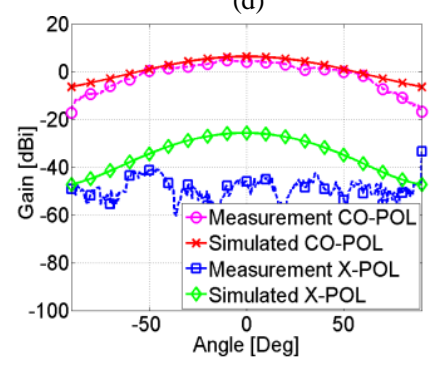

(f)
Fig.8. Simulation and Measurement radiation patterns (a) E-plane at $8 \mathrm{GHz}$ (b) $\mathrm{H}$-plane at $8 \mathrm{GHz}$ (c) E-plane at $10 \mathrm{GHz}$ (d) H-plane at $10 \mathrm{GHz}$ (e) E-plane at $12 \mathrm{GHz}$ (f) $\mathrm{H}$-plane at $12 \mathrm{GHz}$

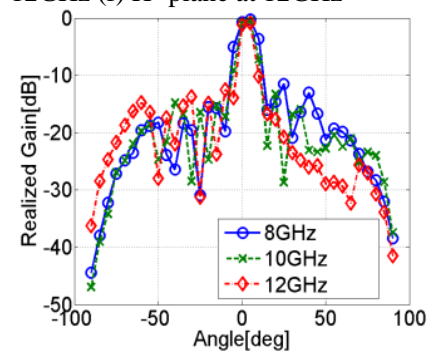

(a)

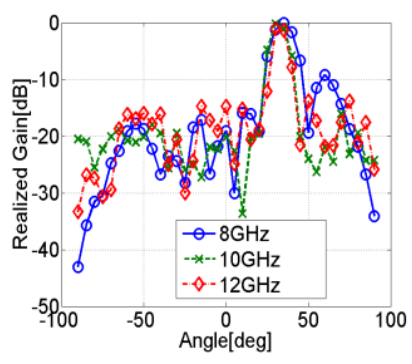

(b)
Fig.9. Normalized Radiation pattern of the antenna array at 8,10,12GHZ: (a) $\Theta=0$ (b) $\Theta=30^{\circ}$

\section{CONCLUSION}

In this letter, a novel type of wideband low-profile all-metal cavity slot antenna with filtering performance for space-borne $\mathrm{SAR}$ application is presented. The antenna structure is formed by a rectangular cavity, a metal block, C-shaped, and H-shaped slots. The $\mathrm{C}$-shaped and $\mathrm{H}$-shaped slots control the filtering performance of the antenna. The antenna shows a simple structure and very low profile $(0.067 \lambda$ at the lowest operating frequency). The multi-element antenna array is made by the metal material, such as aluminium, achieves good heat dissipation and high strength. The measurement results show that antenna has good performance, achieving bandwidth from 7.2-12.2 GHz, about 51\%, and has an average 5dBi realized the gain and more than $20 \mathrm{~dB}$ suppression level between stopband. The array is also shown 30-degree phase scanning ability with good performance.

\section{REFERENCES}

[1] J. Uher, "Comparison of RadarSat-1 and RadarSat-2 SAR antenna design and capabilities," $9^{\text {th }}$ International Symposium on Antenna Technology and Applied Electromagnetics, July. 2002, pp.1-4.

[2] C. G. M.van't Klooster, "On Space-based Synthetic Aperture Radar Antennas," $7^{\text {th }}$ European Conference on Synthetic Aperture Radar, June. 2008, pp.1-4

[3] W. Pitz and D. Miller, "The Terra SAR satellite," IEEE Trans. On Geoscience and Remote Sensing, vol.48, no.2, pp.615-622, Feb.2010.

[4] P. Capece, L. Borgarelli, M. D. Lazzaro, U. D. Marcantonio and A.Torre, "Cosmo-SkyMed active phased array SAR instrument," IEEE Radar Conference, 2008, pp.1-4.

[5] N. Chahat, B. Cook, H. Lim and P. Estabroo, "All-Metal Dual-Frequency RHCP High-Gain Antenna for a Potential Europa Lander" IEEE Trans. On Antennas Propag., vol.66, no.12, pp.6791-6798, Dec. 2018.

[6] V. Mrstik, "Agile-beam synthetic aperture radar opportunities," IEEE Trans.on Aerospace and Electronic systems, vol.34, no.2, pp.500-507, April. 1998.

[7] J. H. G. Ender and A. R. Brenner, "Pamir- a wideband phased array SAR/MTI system," IEE Proc.Radar Sonar Navig., vol.150, no.3, pp. 165172, June. 2003.

[8] C. Mao, Steven. Gao, Y. Wang, F. Qin and Q. Chu, "Multimode Resonator-Fed Dual-Polarized Antenna Array with Enhanced Bandwidth and Selectivity" IEEE Trans. On Antennas Propag., vol.63, no.12, pp.5492-5499, Dec. 2015.

[9] Z. Zheng, W. Wang, H. T. Zhang, M. P. Jin, X. L. Liang, "Waveguide Slot Filtering Antenna Using MushroomType Surface" IEEE Antennas Propag. (AP-S) Int. Symp., Boston, USA, 8-13, July, 2018, PP.745-746.

[10] J. Lu, H. Zhang, W. Wang, X. Liang, and et al, "Broadband dual-polarized waveguide slot filtenna array with low cross-polarization and high-efficiency," IEEE Trans. On Antennas Propag., accepted.

[11] H. Chu, C. Jin, J. X. Chen, and Y. X. Guo, "A 3-D millimeter-wave filtering antenna with high selectivity and low crow-polarization," IEEE Tans. Antennas Propag., vol. 63, no. 5, pp. 2375-2380, Oct. 2015.

[12] W. Duan, X. Y. Zhang, Y.M. Pan, J.X. Xu, and Q. Xue. "Dual-Polarized Filtering Antenna with High Selectivity and Low Cross Polarization" IEEE Trans. Antennas Propag., vol. 64, no. 10, pp. 4188-4196, Oct. 2016.

[13] X. Y. Zhang, W. Duan, and Y.M. Pan, "High-Gain Filtering Patch Antenna Without Extra Circuit" IEEE Trans. Antennas Propag., vol. 63, no. 12, pp. 5883-5888, Dec. 2017.

[14] P. K. Li, C. J. You, H. F. Yu and et al, "Codesigned High-Efficiency Single-Layered Substrate Integrated Waveguide Filtering Antenna with a Controllable Radiation Null", IEEE Antennas Wireless Propag. Lett., vol. 17, no. 2, pp. 295-298, Feb. 2018

[15] L.C. Sun, W. Wang, W. Guan, "A Novel Wideband Metal Cavity Antenna," Radar Science and Technology, vol. 15, no.4, pp:439-448, Aug. 2017.

[16] L.C. Sun, W. Wang, H.T. Zhang, M. P. Jin, and Z.H. Zhang, "Wideband Metal Cavity Antenna", China Patent, CN 201410514936.6. 